\title{
Proposal of High Precise Touch Switch Whose Shape and Placement Can Be Changed Arbitrarily
}

\author{
Kazuki Matsumoto*, Shun Inoue, Seiichi Serikawa \\ Department of Electrical Engineering and Electronics, Kyushu Institute of Technology, Fukuoka, Japan \\ *Corresponding Author. matsumoto@ boss.ecs.kyutech.ac.jp
}

\begin{abstract}
Unlike a conventional mechanical button, there are capacitive touch switches to which the human touches by finger. Since their shape is decided by a maker, we cannot reform their shape to fit to a spherical or irregularity surface. Typically, the placement of them is limited to a flat plane. That is, if the shape of touch switch and the number of switches can be rearranged after shipment, such touch switches may be useful for the users to establish their own product. We have developed such touch switches by using a metal film. The metal film can be cut as arbitral shape and put to any place. This paper proposes new type of touch switch with higher accuracy compared with a conventional touch switches we have proposed. Instead of conventional touch switches, new touch switch can eliminate the effect of the register error. In addition, the structure of touch switches is simpler than the conventional one. Through a comparative experiments, we show that the proposed switch improve the voltage linearity used for detecting a touched switch.
\end{abstract}

Keywords: touch switch, metal film, capacitive, shape, placement

\section{Introduction}

Unlike a conventional mechanical button, there are capacitive touch switches to which the human touches by finger. These touch switches are used for an automatic door or a household appliance. Since their shape is decided by a maker, we cannot reform their shape to fit to a spherical or irregularity surface.

Recently, a bent electrostatic touch switch ${ }^{[2]}$ is appearing. This product can use even on a spherical surface and an irregularity side as a touch switch. This characteristic can be achieved by using a thin electrode film sheet. However, the shape of the touch switch cannot be changed. Also, it is very expensive yet.

To tackle these problems, we have proposed a touch switches by using a metal film ${ }^{[1]}$. The metal film can be cut as arbitral shape and put to any place. However, this touch switch has the problem that the number of switches cannot be expanded easily because the linearity of voltage differences over the used switches used that decides which switch is pushed is not good.

This paper proposes new type of touch switch with higher accuracy compared with the conventional touch switch we have proposed. Instead of the conventional touch switch, new touch switch can improve the accuracy by improving the linearity of voltage differences over the used switches when a switch is touched. In addition, the structure of touch switches is simpler than the conventional one. Through a comparative experiments, we show that the proposed switch improve the voltage linearity used for detecting a touched switch.

\section{Proposed System}

\subsection{System organization of this study}

We show the system organization of this study in figure 1 . We use a high-frequency component from an oscillation circuit. This circuit detects to use electric current $i_{1}, i_{2}$ of the detecting element part. Then, electric current ratio can be calculated as Eq. 1. Electric current ratio thereby changes, and the identification of the touch switch is enabled by the difference electric current when a finger touches the switch part of the touch switch because an electric current flows through the ground.

$$
\text { rate }=\frac{i 1-i 2}{i 1+i 2}
$$




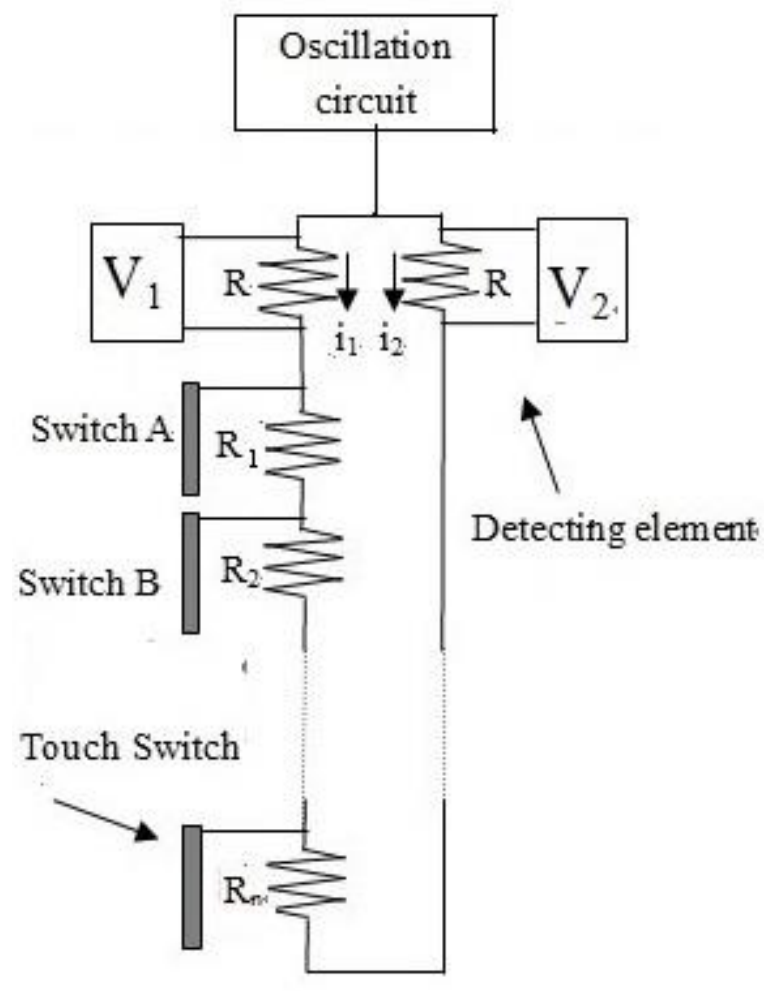

Fig 1. Touch Switch Organization

When he/she touches a switch, a sequence circuit of a capacitor and a resistor to the ground through the human body is made. At first, since $V_{1}$ and $V_{2}$ are same, the current is not found. By touching a switch, the loop circuit is divided at the touched switch that makes the sequence circuit of the capacitor and resistor. As a result, $\mathrm{V}_{1}$ and $\mathrm{V}_{2}$ become different voltage, and the current flows $\left(i_{1}\right.$ and $i_{2}$ ) appear.

\subsection{System organization of the precedent study ${ }^{[1]}$}

We use the conventional touch switch we have proposed as a comparison to show that new type of touch switch has higher precision than the conventional one.

We show the touch switch organization of conventional one in figure 2. This circuit detects the switches touched by using the similar method mentioned above. However, this circuit uses the bridge circuit made when the human touches a switch.

Since the synthesized resistor from the bridge circuit made by human touch is used, the resistor error can be inserted easily compared with the new type of the touch switch. Also, its structure is obviously more complex than the new type of touch switch.

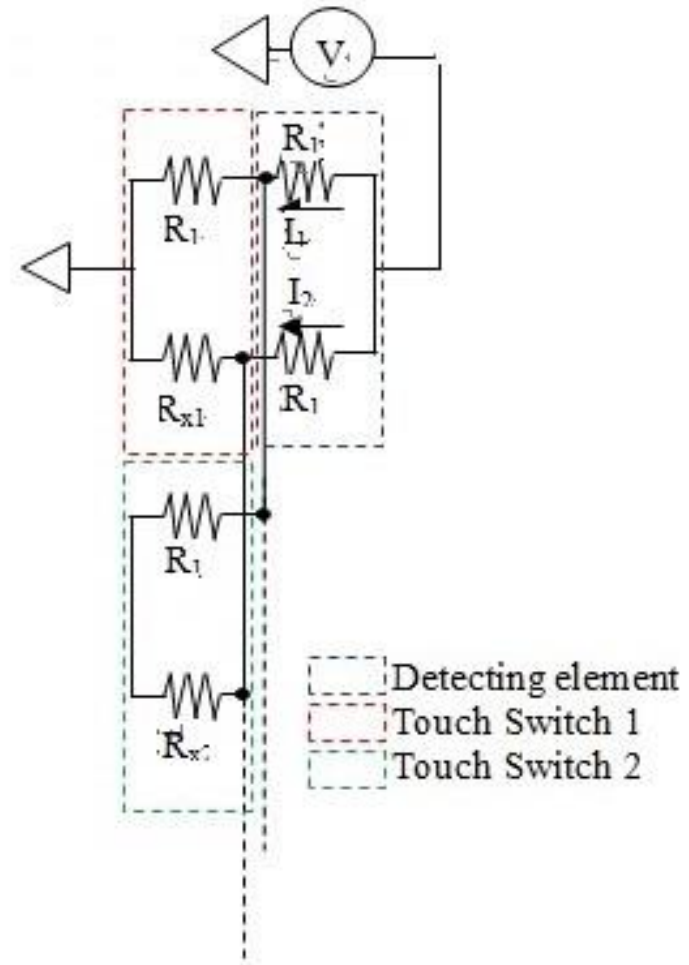

Fig .2. Conventional Touch Switch

\section{Experiment method}

\subsection{Circuit Parameters of New Touch Switch}

In this experiment, we measured the electric current ratio in case of the two from five switches. We use an aluminum foil as a switch part.

We use a sine wave of $1 \mathrm{MHz}$ from a function generator IC as a power supply.

We used a value of $R=1[\mathrm{k} \Omega]$ as resistance of figure 1 and measured the voltage among this resistor to calculate the electric current ratio. This voltage ratio becomes equal to the electric current ratio.

We assume that touch switches in figure 1 are touch switch A, touch switch B ... from the left.

\subsection{Circuit Parameters of Conventional Touch Switch}

As new touch switch mentioned above, we measured the electric current ratio in case of the two from five switches. We use an aluminum foil as a switch part. We use a sine wave of $1 \mathrm{MHz}$ from a function generator IC as a power supply.

This circuit assumed a combination of resistance of the touch switch $\left(\mathrm{R}_{1}, \mathrm{R}_{\mathrm{X} 1}\right)$. When we assumed a value of resistance $R_{1}=100[\Omega]$, we changed a value of $R_{X}$ with 100 , $180,270,360,470[\Omega]$ and measured the electric current 
ratio. This voltage ratio becomes equal the electric current ratio.

\section{Experiment result}

Figure 3 to Figure 6 show the experiment result in case of five from two switches for the new touch switch. Figure 7 shows the experiment result of the conventional touch switch in five switches.

For new touch switch, we can find the obvious voltage differences up to five touch switches in this experiment. However, the conventional touch switch saturates the voltage difference. Thus we think that it is hard to distinguish the touched switch from others.

The experiment result shows that new touch switch can provide better linearity over switches than that of the conventional one. Thus, we think new touch switch achieve more highly precise than conventional one.

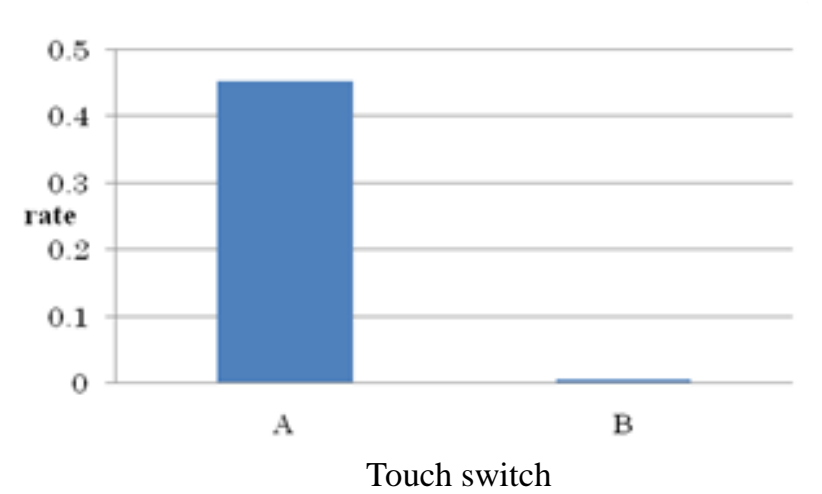

Fig.3. Experiment result of touch switch two

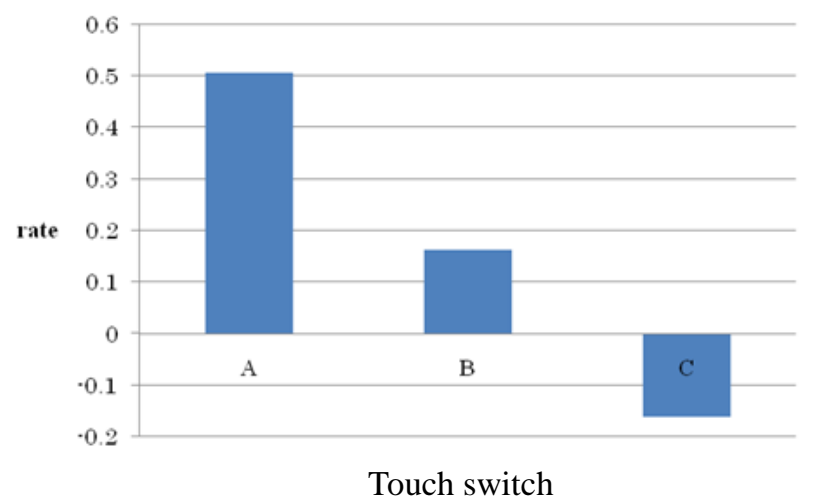

Fig.4. Experiment result of touch switch three

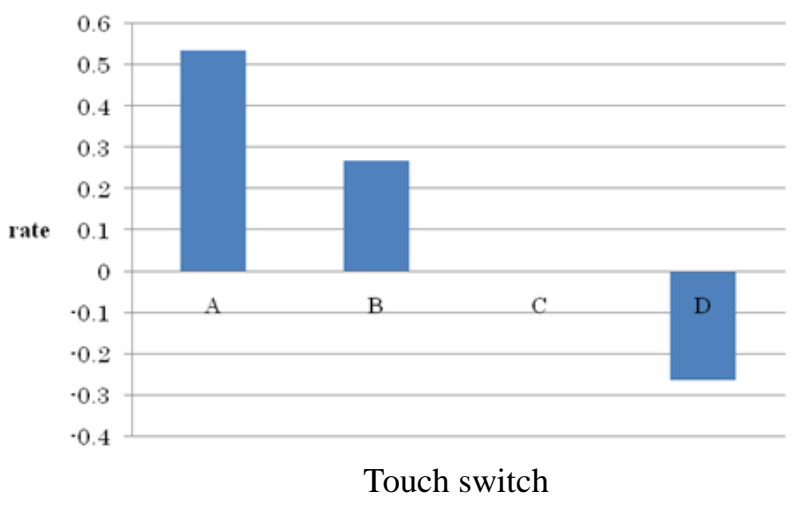

Fig.5. Experiment result of touch switch four

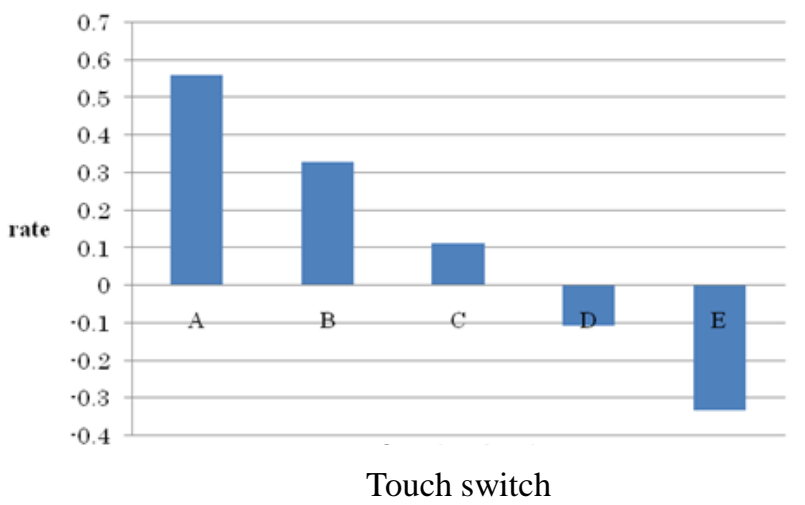

Fig.6. Experiment result of touch switch five

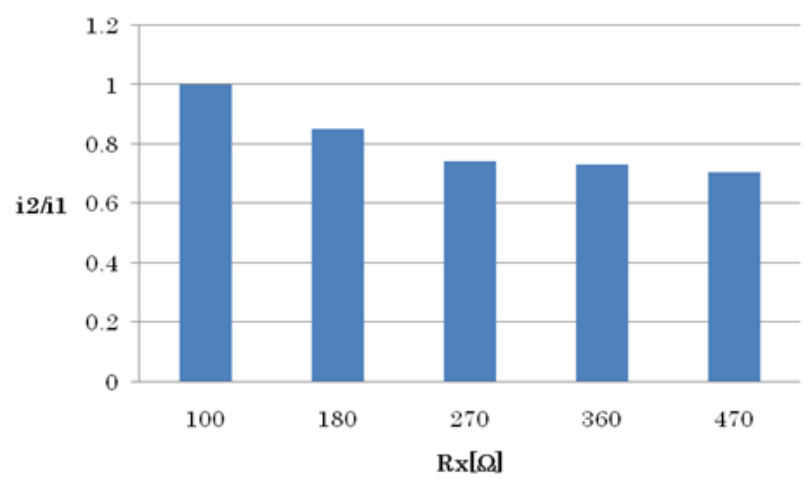

Fig.7. Experiment result of the precedent study

\section{Conclusion}

In this paper, we have shown the highly precise touch switch that a shape and placement are arbitrary. By the difference of the electric current ratio, we have demonstrated the voltage linearity which is used to detect a 
touched switch. Thus, good identification of touch switch is possible.

As future work, we will increase the number of the touch switches more than five and perform the evaluations with many switches. In addition, we will develop real systems that use our touch switches.

\section{References}

[1] Shun Inoue,

"Proposal of a touch switch with arbitrary form and arrangement"

[2] pentel electronic oivision, capasitve switch

http://www.e-pentel.jp/product/touch_switch/

2013.4.1

[3] Assembly Desk, touchsensormodule

http://a-desk.jp/modules/forum_module/index.php?cat_i

$\mathrm{d}=2$

2013.5.10 